\title{
Distribution Network Expansion Planning Considering the Flexibility Value for Distribution System Operator
}

\author{
Ricardo Faia ${ }^{1,2}$ \\ Bruno Canizes ${ }^{1,2}$ \\ Pedro Faria ${ }^{1,2}$ \\ Zita Vale ${ }^{2}$ \\ rfmfa@isep.ipp.pt \\ brmrc@isep.ipp.pt \\ pnf@isep.ipp.pt \\ zav@isep.ipp.pt
}

${ }^{1}$ GECAD - Research Group on Intelligent Engineering and Computing for Advanced Innovation and Development

Rua Dr. António Bernardino de Almeida, 431; 4249-015 Porto; Portugal

${ }^{2}$ Institute of Engineering - Polytechnic of Porto (ISEP/IPP)

Rua Dr. António Bernardino de Almeida, 431; 4249-015 Porto; Portugal

\begin{abstract}
The electric power system has undergone numerous changes over the years. The transformation of the end-users from passive actors to active actors brings implications for the electric power system. The distribution system operator will be able to guide its operations in the function of the active role of the endusers. In many situations, the distribution system operator is carried out to avoid congestion in the distribution networks, and when it happens the distribution system operator is obliged to compensate the affected end-users. This paper presents a model in which distribution system operator can take advantage of the flexibility of the end-users in order to minimize the costs of the investments in distribution network expansion. The investment cost with the presented methodology as show the results has a reduction of $5.77 \%$.
\end{abstract}

Index Terms - Demand Side Flexibility, Distributed Network Planning, Optimal Power Flow

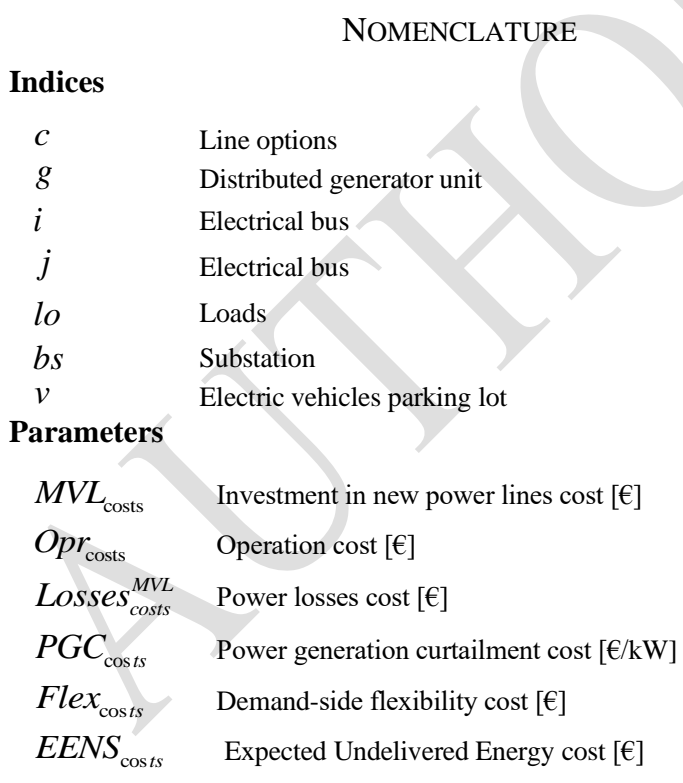

This work has received funding from the European Union's Horizon 2020 research and innovation programme under project DOMINOES (grant agreement No 771066) and from FEDER Funds through COMPETE program and from National Funds through FCT under the project UID/EEA/00760/2019. Ricardo Faia is supported by national funds through Fundação para a Ciência e a Tecnologia (FCT) with PhD grant reference SFRH/BD/133086/2017 and Bruno Canizes is supported by FCT Funds through SFRH/BD/110678/2015 PhD scholarship.

\begin{tabular}{|c|c|}
\hline$p_{D G(g)}$ & Power of distributed generation $g[\mathrm{~kW}]$ \\
\hline Flow $_{(i, j, c)}^{\max }$ & $\begin{array}{l}\text { Maximum admissible line flow between bus } i \text { and bus } j \\
\text { according to the chosen line option } c \text { [MW] }\end{array}$ \\
\hline$n D G$ & Number of DG units \\
\hline$P_{\text {SMinLimit }(b s)}$ & Minimum active power of substation $b s[\mathrm{~kW}]$ \\
\hline$P_{\text {SMaxLimit }(b s)}$ & Maximum active power of substation $b s[\mathrm{~kW}]$ \\
\hline$p_{\text {Load (lo) }}$ & Power demand for load $l o[\mathrm{~kW}]$ \\
\hline$L F_{(i, j, c)}$ & $\begin{array}{l}\text { Loss factor between bus } i \text { and bus } j \text { according to the } \\
\text { chosen line option } c\end{array}$ \\
\hline$P_{\text {ChargeLimit (v) }}$ & Power charge limit for $v \mathrm{EV}$ parking lot $[\mathrm{kW}]$ \\
\hline$P_{\text {LoadFlex }(l o)}^{\max }$ & Demand-side flexibility limit for $l o$ load $[\mathrm{kW}]$ \\
\hline \multicolumn{2}{|l|}{ Variables } \\
\hline $\operatorname{Flow}_{(i, j, c)}$ & $\begin{array}{l}\text { Power flow between bus } i \text { and bus } j \text { according to the } \\
\text { chosen line option } c[\mathrm{~kW}]\end{array}$ \\
\hline$p_{P G C(g)}$ & $\begin{array}{l}\text { Power generation curtailment of distributed generator } g \\
{[\mathrm{~kW}]}\end{array}$ \\
\hline$p_{\text {Supplier }(b s)}$ & Generation power of the substation $b s[\mathrm{~kW}]$ \\
\hline$p_{\text {Charge }(v)}$ & Power charge for $v$ EV parking lot $[\mathrm{kW}]$ \\
\hline$p_{\text {LoadFlex }(l o)}$ & Demand-side flexibility for $l o$ load $[\mathrm{kW}]$ \\
\hline$y_{(i, j, c)}$ & $\begin{array}{l}\text { Binary decision variable }\{0,1\} \text { for the line usage between } \\
\text { bus } i \text { and bus } j \text { according to the chosen line option } c\end{array}$ \\
\hline$u_{(l o)}$ & $\begin{array}{l}\text { Binary decision variable }\{0,1\} \text { for the demand-side } \\
\text { flexibility of load } l o \text { usage }\end{array}$ \\
\hline \multicolumn{2}{|r|}{ Henta } \\
\hline$\Omega_{L}^{b}$ & Set of load buses \\
\hline$\Omega_{V}^{b}$ & Set of EV buses \\
\hline$\Omega_{o}$ & Set of line options \\
\hline$\Omega_{l}$ & Set of lines \\
\hline$\Omega_{B}$ & Set of buses \\
\hline$\Omega_{D G}^{n d}$ & Set of non-dispatchable DG \\
\hline$\Omega_{B S}^{b}$ & Set of substation buses \\
\hline$\Omega_{j}^{b}$ & Set of buses without substation buses \\
\hline
\end{tabular}

Flow $_{(i, j, c)}$

$n D G$

$P_{\text {SMinLimit }(b s)}$

$P_{\text {SMaxLimit }(b s)}$

$p_{\text {Load }(l o)}$

$L F_{(i, j, c)}$

$P_{\text {Chargelimit }(v)}$ $P_{\text {LoadFlex }(l o)}^{\max }$

Variables 


\section{INTRODUCTION}

The intermittent Distributed Energy Resources (DREs), demand-side flexibility and energy storage systems transformed the role of traditional end-users in the electrical power system, transforming them into active users. The demand side flexibility represents the part of the load that can be changed without violating the comfort standards of the users. Demand-side flexibility through Demand Response (DR) in end-users is a balancing mechanism that can be used to suppress problems of intermittent DERs [1]. According to the objectives described in the $3^{\text {rd }}$ package adopted by the European Union, they foresee a minimization of $\mathrm{CO} 2$ emissions and emissions with greenhouse gases [2]. For this reduction to occur, it is necessary to integrate more clean generation in the electric power system.

The clean generation produced from DERs has in the last years a massive integration into the power system. With this integration, new actors and roles are emerging. The prosumers concept is an example of these new actors, which combines consumer, storage and local level generator capabilities. This characteristics of prosumers enable electricity and economic transactions in nowadays called local electricity markets [3]. The energy exchange scenario in the future is moving to several local markets bounded by geographic space. According to Smart Energy Collective alliance, an aggregator's role consists of accumulating flexibility in active demand and supply [4]. The flexibility costumers are defined by Universal Smart Energy Framework [5] as four different entities: the Distribution System Operator (DSO), Balance Responsible Parties (BRP), Transmission System Operator (TSO) and Prosumers. DSO and TSO are interesting to obtain flexibility to manage grid congestion and reduce the cost of the investment. BRP should use the flexibility to organize its portfolio and reduce operating costs. For the Prosumers can use the flexibility capabilities to reduce their electricity bill.

The aggregators in power systems have an important social role, they have the task of bringing the final customers closer to entities such as the DSO, TSO and in markets such as the Spot market. The Aggregators as previous said, they collect the flexibility from the end user and sell for customers. In reference [6] the authors present the demand response aggregators (DRAs) to integrate the power flexibility of the contracted costumers. The DRAs's aim maximization the profit of the end users in the energy market and ancillary services market. The DR has a useful instrument for DSO and TSO, because it is a source of flexibility, for aggregator is good because it can do your own business around it. For end users is also a useful instrument because as the reference [7] demonstrates the daily costs operations can be reduced with the use of DR.

Both TSO and DSO could benefit from the use of flexibility resources from the distribution grid, but is not easy for TSO and DSO to make use of theses flexibility services under the liberalization regime [8]. This paper proposes an analysis of the value that the flexibility available in end-users can bring to the DSO for management of distribution network. A case study with 13 buses network is present, all of the buses are in medium voltage and the connected loads is considering an aggregated of loads. With the methodology presented, it is intended to identify the most viable solution between investing in network reinforcement or/and using the flexibility available in endusers. This paper is structured into five sections, the first section presents an introduction, in section number two is presented the proposed methodology the case study is presented in the third section. Results and conclusions are presented in section number four and five respectively.

\section{PROPOSED MethodOLOGY}

Flexibility in traditional electric power systems was achieved by the generation side, by the re-dispatch of the units and the start-up of auxiliary generation units. With the increase of DERs from Renewable Energy Sources (RESs) the flexibility has become more difficult to obtain due to the intermittent character of these RES. Nowadays the planning of the distribution networks by the DSO will have to rely on this RES intermittencies. RES are mostly DERs and are installed very close to consumption points. The demand side flexibility that is obtained from the consumer's side may be an option to be taken into account by the DSO in the planning of the distribution networks, thus being able to minimize the costs incurred in the physical expansion of the network. For DSO to be able to use demand-side flexibility in its distribution networks planning actions, the users will have to make it available. Getting users to provide flexibility when required is a challenging and crucial topic that is currently in a position of debate among regulators, DSOs, customers and DER suppliers [9].

In this paper propose a model, based on DC optimal power flow presented in reference [10], that can be used as a decisionsupport tool to assist the DSO in the planning of future investments in the distribution network making use of the flexibility of the demand side. The DC optimal power flow is used to reduce the complexity and computational burden of the model, improving its tractability.

As a consequence, the voltage magnitude is one p.u. and the reactive power flow is neglected. On the other hand, MV distribution networks in several countries like Portugal have transformer with automatic voltage regulators and capacitor banks carefully placed along the network to keep the voltage and reactive power between the desired limits. Thus, using a complete model for the long-term planning problem (i.e., AC optimal power flow) would only make the method more complex and computationally heavy. [11]. In this model, as can be observed by the objective function Eq. (1), the objective is to minimize investment costs in new lines and operating costs.

$$
M i n T_{\text {costs }}=M V L_{\text {costs }}+O p r_{\text {costs }}
$$

The operational costs in Eq. (2) consider the costs of energy losses in medium voltage lines Losses $_{\text {costs }}^{M V L}$, the power generation curtailment $P G C_{\text {costs }}$, the expected undelivered energy $E E N S_{\text {costs }}$ and the flexibility uses costs Flex $x_{\text {costs. }}$.

$$
O p r_{\text {costs }}=\text { Losses }_{\text {costs }}^{M V L}+P G C_{\text {costs }}+\text { EENS }_{\cos t s}+\text { Flex }_{\text {costs }}
$$

The Eq. (3) represents the power balance constraint which is checked for all buses. 


$$
\begin{aligned}
& \sum_{i \in \Omega_{D G}^{n d}}\left(p_{D G(i)}-p_{P G C(i)}\right)+\sum_{i \in \Omega_{B S}^{b}} p_{\text {Supplier }(i)}+ \\
& \sum_{i \in \Omega_{L}^{b}} p_{\text {LoadFlex }(i)}-\sum_{i \in \Omega_{L}^{b}} p_{\text {Load }(i)}-\sum_{i \in \Omega_{V}^{b}} p_{\text {Charge }(i)}- \\
& \sum_{i \in \Omega_{B}} \sum_{c \in \Omega_{O}}\left(\operatorname{Flow}_{(i, j, c)} \cdot L F_{(i, j, c)}\right)+ \\
& \sum_{i \in \Omega_{B}} \sum_{c \in \Omega_{O}} \operatorname{Flow}_{(i, j, c)}-\sum_{i \in \Omega_{B}} \sum_{c \in \Omega_{O}} \operatorname{Flow}_{(j, i, c)}=0 \\
& \forall j \in \Omega_{B}
\end{aligned}
$$

The power flow limit in each line is modeled in Eq. (4).

$$
\begin{aligned}
& 0 \leq \text { Flow }_{(i, j, c)} \leq \text { Flow }_{(i, j, c)}^{\max } \cdot y_{(i, j, c)} \\
& \forall(i, j, c) \in \Omega_{l}, \forall y \in\{0,1\}
\end{aligned}
$$

Constraint Eq. (5) guarantees unidirectionality between bus $i$ and $j$ and also the choice of only one line type $c$ in that direction.

$$
\begin{aligned}
& y_{(i, j, c)}+y_{(j, i, c)} \leq 1 \\
& \forall y \in\{0,1\}, \forall(i, j, c) \in \Omega_{l}
\end{aligned}
$$

Only one line type $c$ can be chosen for each line location (Eq. (6)).

$$
\begin{aligned}
& \sum_{c \in \Omega_{o}} y_{(i, j, c)} \leq 1 \\
& \forall y \in\{0,1\}, \forall(i, j, c) \in \Omega_{l}
\end{aligned}
$$

To ensure the radial topology, constraint (Eq. (7)) is used. This constraint imposes that only one line can enter in each bus.

$$
\begin{aligned}
& \sum_{j \in \Omega_{L}^{b}} \sum_{c \in \Omega_{O}} y_{(i, j, c)}=1 \\
& y \in\{0,1\}, \forall i \in \Omega_{B}
\end{aligned}
$$

To avoid DG isolation from the substation, the constraints Eq. Error! Reference source not found.-(11) are applied to the mathematical model. To do this, it is created a fictitious flow $\left(d_{(i, j, c)}\right)$ with a fictitious load of each DG $\left(D_{(g)}\right)$ to be fed by the substation. If the island is permitted, the planner can omit these equations.

$$
\begin{gathered}
\sum_{i \in \Omega_{B}} \sum_{j \in \Omega_{B}} \sum_{c \in \Omega_{O}} d_{(i, j, c)}-\sum_{i \in \Omega_{B}} \sum_{j \in \Omega_{B}} \sum_{c \in \Omega_{O}} d_{(j, i, c)}=D_{(g)} \\
\forall g \in \Omega_{D G} \\
D_{(g)}=1 \\
\forall g \in \Omega_{D G} \\
D_{(g)}=0 \\
\forall g \notin \Omega_{D G} \cup \Omega_{B S}
\end{gathered}
$$

$$
\begin{aligned}
& \left|d_{(i, j, c)}\right| \leq n D G \cdot y_{(i, j, c)} \\
& \forall(i, j, c) \in \Omega_{l}
\end{aligned}
$$

The power supplied by a substation is constrained by the maximum and minimum capacity that can supply (Eq. (12)).

$$
\begin{aligned}
& P_{\text {SMinLimit }(b s)} \leq p_{\text {Supplier }(b s)} \leq P_{\text {SMaxLimit }(b s)} \\
& \forall b s \in \Omega_{B S}
\end{aligned}
$$

The electrical vehicles (EVs) are considered as parking lots or a set of EVs located in the network. The parking lot charge is equal to charge limit multiplied by simultaneity factor $(s f)$. The charge limit for each parking lot $v$ is modeled in Eq. (13).

$$
\begin{aligned}
& p_{\text {Charge }(v)}=P_{\text {ChargeLimit }(v)} \cdot s f \\
& \forall v \in \Omega_{V}^{b}
\end{aligned}
$$

The power generation curtailment is verified when the excess of a generation of generator $g$ occurs. This variable is lower or equal to the generation of $g$ generator (Eq. (14)).

$$
\begin{aligned}
& 0 \leq p_{G C P(g)} \leq p_{D G(g)} \\
& \forall g \in \Omega_{D G}^{n d}
\end{aligned}
$$

Demand-side flexibility is given by Eq. (15).

$$
\begin{aligned}
& 0 \leq p_{\text {LoadFlex }(l o)} \leq P_{\text {LoadFlex }(l o)}^{\max } \cdot u_{(l o)} \\
& \forall l o \in \Omega_{L}^{b}, \forall u \in\{0,1\}
\end{aligned}
$$

\section{CASE STUDY}

In this work, as was said is proposed a model that can be used as a decision-support tool to assist the DSO in the planning of future investments in the distribution network making use of the demand side flexibility. In this way is considering 10 years for lifetime project, the investments are all carried out at the beginning of the project. For costs of energy not supply is considered $3 € / \mathrm{kWh}$ and $0.12 € / \mathrm{kWh}$ for losses price. The authors assume that the DSO can only request one cut event per year with 1 hour of duration, and in the 10-year lifetime project, it can order 10 events. A cut event represents the cut of load in a certain period with a certain duration, which is usually used to avoid problems in the management of the network. For simulations studies are considered $0.05 \%$ of the discount rate.

In Fig. 1 is presented the network used for the study case. As it can be observed the distribution network has 14 buses in medium voltage level $(30 \mathrm{kV})$. Connections between buses are made by AA 90 overhead lines type. The network presented different load points, each load point considers an aggregation of several end-users. The network has also connected RES such as wind farm and solar panel. An EV parking lot is also presented. The lines that are presented in dashed lines correspond to the possible new investments. This network was constructed in mesh topology, but its operation is carried out in radial topology (constraint of eq. (7)).

As Fig. 1 present, a network with nine load points, one EV parking lot, one wind farm, two solar panels and in buses, number 1 is connected the substation. The substation has a production capacity of $30 \mathrm{MW}$, photovoltaic and wind utilities 
have $0.75 \mathrm{MW}$ for maximum capacity, and the parking lot have $1.20 \mathrm{MW}$ of maximum capacity. The total load points have aggregated 10.3 MW of consumed capacity.

In this case study, is considered investing in new lines construction and investing in contacts of flexibility reduction capacity for ten years. For new lines of investment, two option is considered. Option 1 (line AA90) and option 2 (line AA160) respectively [12]. In the actual network, the lines are AA90 type. This method also considers the possibility to change a line type for the other (AA90 by AA160). Taking into account the investments in contracts of flexibility reduction capacity is considered three different price values for energy contracted, $0.10,0.15$ and $0.20 € / \mathrm{kW}$. Is also considered three different values for total cut power in network load points, $0.25,0.50$ and $0.75 \mathrm{MW}$.

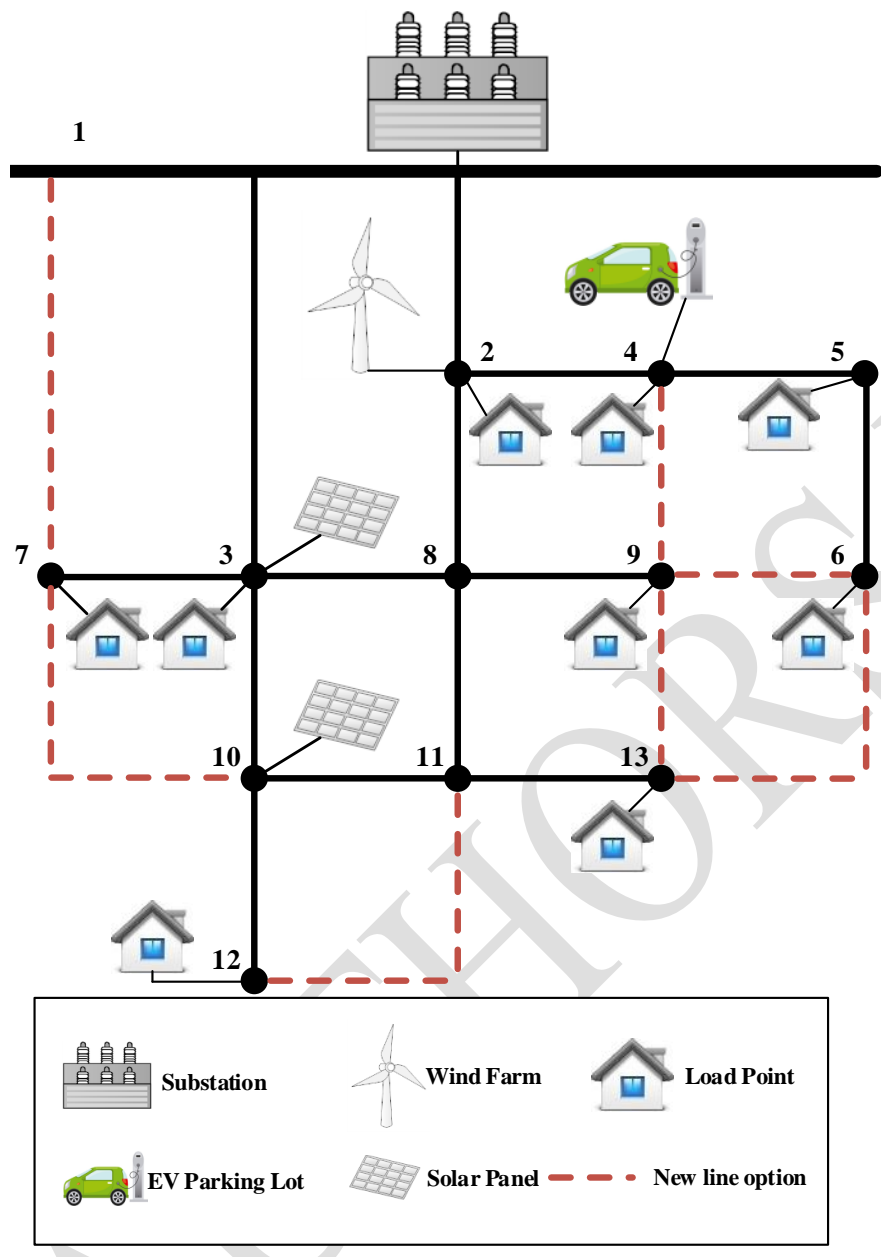

Fig. 1. 13 buses network example for the test (adapted from [10])

With the values presented above about price for energy contracted and total cut power is possible to create nine different combinations. In the results section the nine different results combinations are present, in order to compare results a is present also a base case. The base case is only considered the investments in new lines construction without investments in contacts for flexibility reduction capacity. It should be noted that usually the distribution networks are designed meshed, but it is operated radially [11].
The proposed work was developed in MATLAB R2018a and TOMLAB 8.164 bits with CPLEX solver (version 12.5) using a computer with one Intel Xeon E5-2620 v2 processor and 16 GB of RAM running Windows 10 Pro.

\section{RESULTS}

In this section, the results are shown. TTABLE 1 presents the results for each combination considering the cut power and the price for $\mathrm{kW}$ cut. In each combination is presented the results for investments costs in new lines, the lines losses costs, the costs of energy not supplied, the costs of flexibility contracts reduction and the total costs of planning option. In TABLE 1 are present the results in $€$ for each combination. The total costs increase with the price applied to the $\mathrm{kW}$ cut but decrease with the cut power increment.

TABLE 1 RESULTS FOR EACH COMBINATION $(€)$

\begin{tabular}{|c|c|c|c|c|}
\hline \multirow{2}{*}{$\begin{array}{c}\begin{array}{c}\text { Cut } \\
\text { power } \\
(\mathbf{k W})\end{array} \\
\end{array}$} & \multirow{2}{*}{ Parameter } & \multicolumn{3}{|c|}{ Price per kW cut } \\
\hline & & 0.10 & 0.15 & 0.20 \\
\hline \multirow{5}{*}{250} & $M V L_{\text {costs }}$ & 816752.87 & 816752.87 & 816752.87 \\
\hline & Losses ${ }_{\text {costs }}^{M V L}$ & 1400548.47 & 1400548.47 & 1400548.47 \\
\hline & $E N S_{\cos t s}$ & 1595214.91 & 1595214.91 & 1595214.91 \\
\hline & Flex $x_{\cos t s}$ & 249.31 & 373.97 & 498.63 \\
\hline & $T_{\text {costs }}$ & 3812765.57 & 3812890.22 & 3813014.88 \\
\hline \multirow{5}{*}{500} & $M V L_{\text {costs }}$ & 816752.87 & 816752.87 & 816752.87 \\
\hline & Losses ${ }_{\text {costs }}^{M V L}$ & 1366080.81 & 1366080.81 & 1366080.81 \\
\hline & $E N S_{\cos t s}$ & 1554570.76 & 1554570.76 & 1554570.76 \\
\hline & Flex $_{\cos t s}$ & 498.63 & 747.94 & 997.26 \\
\hline & $T_{\text {costs }}$ & 3737903.06 & 3738152.38 & 3738401.69 \\
\hline \multirow{5}{*}{750} & $M V L_{\text {costs }}$ & 816752.87 & 816752.87 & 816752.87 \\
\hline & Losses $_{\text {costs }}^{M V L}$ & 1331613.15 & 1331613.15 & 1331613.15 \\
\hline & $E N S_{\cos t s}$ & 1513926.60 & 1513926.60 & 1513926.60 \\
\hline & Flex $_{\cos t s}$ & 747.94 & 1121.91 & 1495.88 \\
\hline & $T_{\text {costs }}$ & 3663040.56 & 3663414.53 & 3663788.50 \\
\hline
\end{tabular}

In TABLE 1, analyzing the costs of flexibility reduction contracts, it increases with the price and with the cut power. The investment costs in medium voltage lines are equal in all cases, this means that the investments are always the same. In this cost are considered the costs of investing in new lines and the maintenance costs for 10 years of a lifetime project. The cost of losses in medium voltage lines and the cost of expected undelivered energy have the same behaviour. They decrease with the cut power. TTABLE 2 is present a comparison between the base case and the results for the combination of 750 $\mathrm{kW}$ and $0.15 € / \mathrm{kW}$. The $750 \mathrm{~kW}$ represents the scenario with more DR potential and the price $0.15 € / \mathrm{kW}$ is the average of the present DR cut costs.

Analyzing the values present in TABLE 2 is possible to conclude that brings advantages to the use of flexibility for DSO in distribution network planning. Although the combination of values $(750 \mathrm{~kW}$ and $0.15 € / \mathrm{kW})$ is not the one 
with the lowest total cost value, was chosen for comparison due to the cut price value. Considering the investments in new lines there is no difference in values. In terms of losses and expected undelivered energy there is a reduction of $7 \%$.

TABLE 2 COMPARISION OF RESULTS

\begin{tabular}{|c|c|c|c|c|c|}
\hline \multirow[b]{2}{*}{ Scenarios } & \multicolumn{2}{|c|}{ Investment ( $€$ ) } & \multicolumn{2}{|l|}{ Charges } & \multirow[b]{2}{*}{ Total costs } \\
\hline & Lines & \begin{tabular}{|l|} 
Flexibility \\
contracts
\end{tabular} & Losses & EENS & \\
\hline Base case & 816752 & 0 & 1435016.14 & 1635859.06 & 3887628.07 \\
\hline $\begin{array}{r}750 \mathrm{~kW} \\
0.15 € / \mathrm{kW} \\
\end{array}$ & 816752 & 1121 & 1331613.15 & 1513926.60 & 3663414.53 \\
\hline \multicolumn{3}{|r|}{ Difference $(€)$} & 103402.99 & 121932.46 & 224213.54 \\
\hline \multicolumn{3}{|r|}{ Reduction (\%) } & $7.21 \%$ & $7.45 \%$ & $5.77 \%$ \\
\hline
\end{tabular}

In view of the total costs, the use of cut power contracts in distribution network planning causes a decrease of 5,77\% related to base case total costs. In Fig. 2 is represented the network reconfigured considering the results with the use of $750 \mathrm{~kW}$ for cut power and $0.15 € / \mathrm{kW}$ for the price cut.

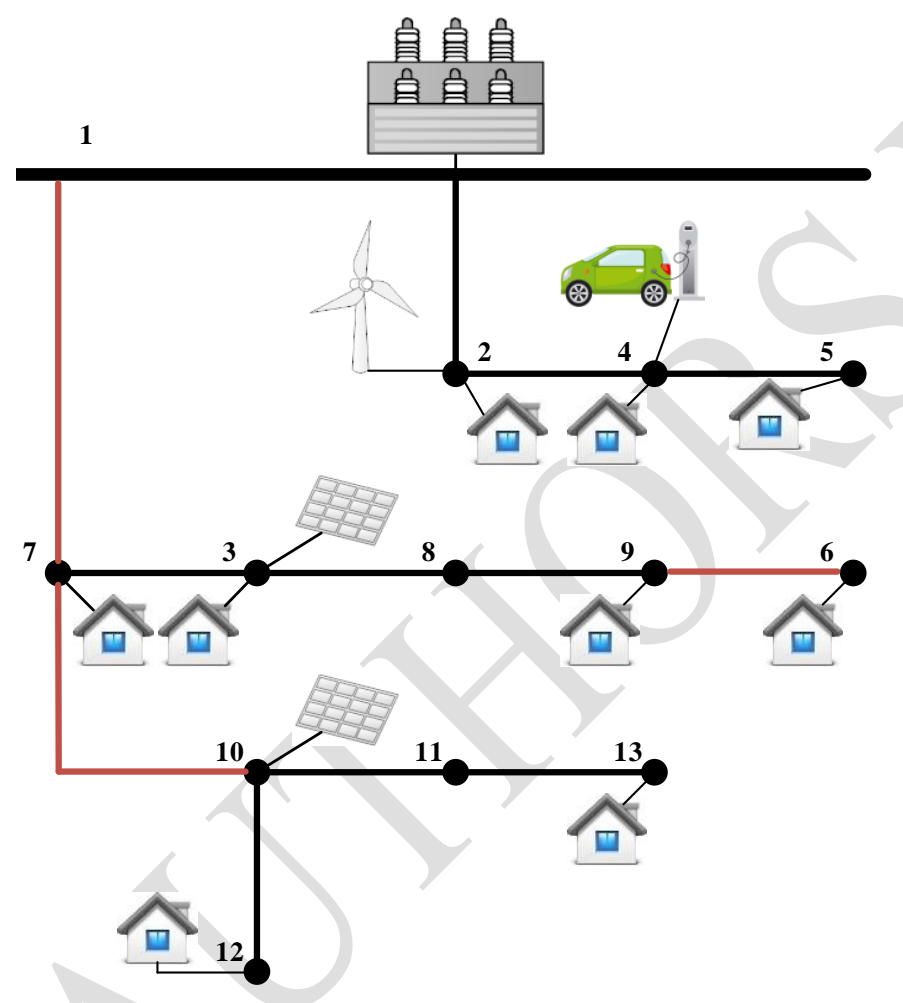

Fig. 2. 13 buses network reconfigured

Analyzing the reconfiguration of network present in Fig. 2 there are three new lines. The lines of buses 1 to 3,2 to 8,5 to 6,3 to 10 and 8 to 11 they are no longer need, physically they are installed, but for this case they are not used. In TABLE 3 is present the power flow results for scenarios with the base case and $750 \mathrm{~kW}$ and $0.15 € / \mathrm{kW}$.

TABLE 3 POWER FLOW ANALYSES (KW)

\begin{tabular}{|r|r|r|r|r|}
\hline \multirow{2}{*}{ Bus in } & \multirow{2}{*}{ Bus out } & \multicolumn{2}{|c|}{ Power flow (kW) } & Reduction (\%) \\
\cline { 3 - 5 } & & Base case & $\mathbf{7 5 0} \mathbf{~ k W ~ 0 , 1 5} \mathbf{\epsilon} / \mathbf{k W}$ & \\
\hline 1 & 2 & 2732 & 2496 & 0,09 \\
\hline 1 & 7 & 7276 & 6761 & 0,07 \\
\hline 2 & 4 & 2615 & 2425 & 0,07 \\
\hline 3 & 8 & 2078 & 1928 & 0,07 \\
\hline 4 & 5 & 1284 & 1191 & 0,07 \\
\hline 7 & 3 & 4086 & 3840 & 0,06 \\
\hline 7 & 10 & 2033 & 1849 & 0,09 \\
\hline 8 & 9 & 2078 & 1928 & 0,07 \\
\hline 9 & 6 & 771 & 715 & 0,07 \\
\hline 10 & 11 & 848 & 787 & 0,07 \\
\hline 10 & 12 & 1690 & 1568 & 0,07 \\
\hline 11 & 13 & 848 & 787 & 0,07 \\
\hline
\end{tabular}

The flow in the lines has a reduction in the scenario where the power cut is considered. The reduction represents an average of $0.7 \%$ per line in relation to the base case. The TABLE 4 are present the characteristics and costs related to the new lines installed in reconfiguration presented in Fig. 2.

TABLE 4 COST FOR NEW LINES INSTALED BASED ON [12]

\begin{tabular}{|r|r|r|r|r|}
\hline Bus in & Bus out & Line type & Cost (€) & Maintenance costs (€) \\
\hline 1 & 7 & line AA160 & 20250 & 4050 \\
\hline 9 & 6 & line AA90 & 3000 & 600 \\
\hline 7 & 10 & line AA90 & 45000 & 9000 \\
\hline
\end{tabular}

TABLE 4 are present the characteristic of the new lines. In the new three options, one of them is on line AA160, and the two others are line AA90. The line of busses 7 to 10 has higher cost values because of its high length. In fact, the maintenance costs represent $20 \%$ of the total cost. In TABLE 5 presents the results related to the economic analysis considering 10 years for lifetime project.

TABLE 5 ECONOMIC ANALYSIS

\begin{tabular}{|c|c|}
\hline Economic Index & $\mathbf{7 5 0} \mathbf{~ k W}$ and $\mathbf{0 . 1 5} \boldsymbol{\epsilon} / \mathbf{k W}$ \\
\hline Payback (years) & 5.47 \\
\hline IRR $(\%)$ & 12.78 \\
\hline NPV $(€)$ & 224099.24 \\
\hline
\end{tabular}

In TABLE 5, it is possible to see a considerable improvement in the three economic indexes when it is considered the cut power of $750 \mathrm{~kW}$ and $0.15 € / \mathrm{kW}$. The value of 5.47 year for payback means that the investment will be paid after 5 years and 6 months, that is, in half of the life lifetime project. The IRR represents the internal rate of return and gives the rate of growth a project is expected to generate. The value of $12.78 \%$ is a good index for IRR.

The NPV represents a net present value and is used to analyze the profitability of a projected investment or project. With $224099.24 €$ for NPV indicates that the projected 
earnings generated by an investment exceed the anticipated costs. Fig. 3 presents the power flow in each load point.

In the Fig. 3 is presented the power flow in each load point for two different scenarios, the base case and $750 \mathrm{~kW}$ and 0.15 $€ / \mathrm{kW}$. Bus 1 have all load accumulated and is the reason that in the figure have the highest value. It is remarkable in all buses that in the scenario with $750 \mathrm{~kW}$ and $0.15 € / \mathrm{kW}$ the load accumulated in the buses is smaller.

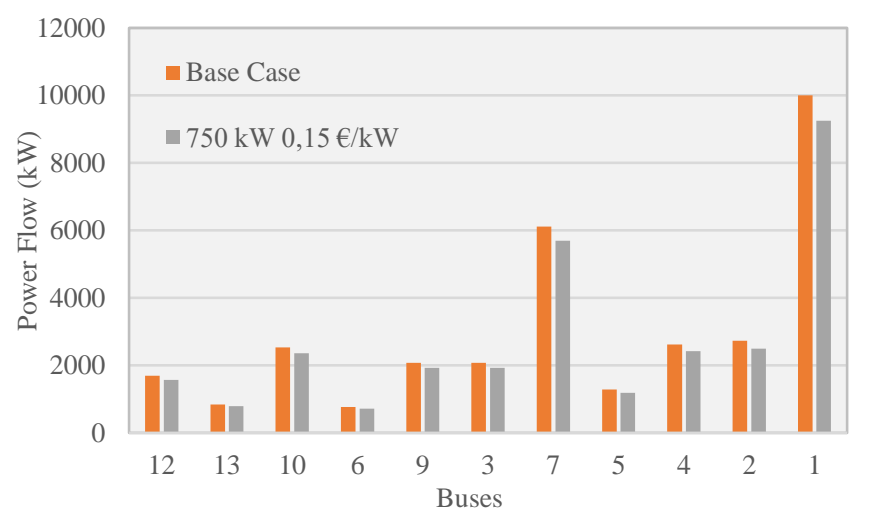

Fig. 3. Power flow analysis considering the load points

\section{CONCLUSION}

This research paper presented a decision-support tool to assist the DSO in the planning of future investments in the distribution network. The main feature of this research paper is the inclusion of demand flexibility in the action of planning for the distribution network. In the results of the simulation, the flexibility contracts reduction has a direct influence on the line losses costs and expected undelivered energy cost.

Considering that the investment results in new lines are the same in all scenarios tested, it is concluded that the cut power amount is insufficient to reducing it. As future work is intended to explore the impact of DR contracts on the expansion of low voltage networks.

\section{REFERENCES}

[1] P.-H. Li and S. Pye, "Assessing the benefits of demand-side flexibility in residential and transport sectors from an integrated energy systems perspective," Appl. Energy, vol. 228, pp. 965-979, Oct. 2018.

[2] European Union Directive, "28/EC. htp," eur-lex. Eur. eu/LexUriServ/LexUriServ. do, 2009.

[3] T. Sousa, T. Soares, P. Pinson, F. Moret, T. Baroche, and E. Sorin, "Peer-to-peer and community-based markets: A comprehensive review," Renew. Sustain. Energy Rev., vol. 104, pp. 367-378, Apr. 2019.

[4] M. Volkerts, F. Verheij, and F. Bliek, "An introduction to the universal smart energy framework," Smart Energy Collect. Arnhem, Netherlands, 2013.

[5] USEF Foundation, "The Framework specifications 2015," Usef, pp. 1-195, 2015.

[6] H. Golmohamadi, R. Keypour, B. Bak-Jensen, and J. R. Pillai, “A multi-agent based optimization of residential and industrial demand response aggregators," Int. J. Electr. Power Energy Syst., vol. 107, pp. 472-485, May 2019.

[7] R. Faia, P. Faria, Z. Vale, and J. Spinola, "Demand Response Optimization Using Particle Swarm Algorithm Considering Optimum Battery Energy Storage Schedule in a Residential House," Energies, vol. 12, no. 9. 2019.

[8] A. Ferrante, N. Constantinescu, and J. A. Jackson, "Lines of convergence: R\&D for transmission and distribution: Coordination and the regulatory challenge," IEEE Power Energy Mag., vol. 13, no. 1, pp. 52-59, Jan. 2015.

[9] I. Abdelmotteleb, T. Gómez, J. P. Chaves Ávila, and J. Reneses, "Designing efficient distribution network charges in the context of active customers," Appl. Energy, vol. 210, pp. 815-826, Jan. 2018.

[10] B. Canizes, J. Soares, M. A. F. Ghazvini, C. Silva, Z. Vale, and J. M. Corchado, "Long-Term Smart Grid Planning Under Uncertainty Considering Reliability Indexes," in Operation, Planning, and Analysis of Energy Storage Systems in Smart Energy Hubs, Springer International Publishing, 2018, pp. 297-335.

[11] S. Batista, B. Canizes, A. Oliveira, T. Nogueira, and Z. Vale, "Optimal location of normally open switches in order to minimize power losses in distribution networks," in 2016 IEEE Power and Energy Society General Meeting (PESGM), 2016, pp. 1-5.

[12] “Grupo Cabelta." [Online]. Available: http://svrweb.cabelte.pt/enus/. 